\title{
Electrotonic Coupling Interacts with Intrinsic Properties to Generate Synchronized Activity in Cerebellar Networks of Inhibitory Interneurons
}

\author{
Puah Mann-Metzer and Yosef Yarom \\ Department of Neurobiology, Life Science Institute and Center for Neural Computation, Hebrew University, \\ Jerusalem 91904, Israel
}

Exploring the organization and function of local inhibitory networks is an essential step on the way to understand the principles of brain operation. We show here that molecular layer inhibitory interneurons of the guinea pig cerebellar cortex are organized as local networks, generating synchronous activity. Simultaneous recording from two adjacent interneurons revealed a direct current flow between synchronized pairs of neurons. Blocking inhibitory or excitatory synaptic transmission did not alter the synchronization. The electrotonic coupling coefficient (average 0.1) depended mainly on the input resistance of the postsynaptic cell, indicating a homogenous coupling resistance between different pairs. A presynaptic action potential generated a short, attenuated spikelet in the postsyn- aptic cell. The passive current flow was amplified by voltagedependent intrinsic currents to create a reciprocal interplay between the presynaptic and postsynaptic cells. This interplay results in a time window for synchronization that is wider than expected from the duration of the spikelet. Intracellular staining with biocytin revealed high incidence of dye coupling. Furthermore, the interneurons located superficially in the molecular layer tend to form larger networks compared with the inner interneurons. We propose that weakly coupled inhibitory networks can generate loosely synchronous activity, which results from the interaction of electrical coupling and intrinsic currents.

Key words: cerebellum; electrotonic coupling; synchronization; inhibitory network; inhibitory interneurons; dye coupling
A functionally intact inhibitory system is a prerequisite for the adequate performance of the nervous system and, indeed, elaborate and complex inhibitory networks can be found throughout the CNS. Despite several recent attempts to study inhibitory networks (Deschênes et al., 1985; Bouskila and Dudek, 1993; Michelson and Wong, 1994; Whittington et al., 1995; Alonso et al., 1996; Benardo, 1997; Dickson and Alonso, 1997; Häusser and Clark, 1997; Pouzat and Hestrin, 1997; Kondo and Marty, 1998), little is known about their basic functional organization.

A common finding in many of these studies is that networks of mutually connected inhibitory interneurons exhibit, under various experimental conditions, synchronized activity. Experimental findings, supported by theoretical work, attribute this synchronicity to the mutual inhibitory synapses (Van Vreeswijk and Abbott, 1994; Whittington et al., 1995; Wang and Buzsáki, 1996). However, the involvement of electrical synapses in creating synchronicity has also been suggested for some of these systems (Bouskila and Dudek, 1993; Michelson and Wong, 1994; Traub, 1995; Benardo, 1997). Because the synchronized activity is frequently rhythmic (Deschênes et al., 1985; Michelson and Wong, 1994; Whittington et al., 1995; Benardo, 1997; Dickson and Alonso, 1997), it has been suggested that one role of inhibitory networks is to impose a rhythm on the activity of the principle neurons, providing the context to their operation (Buzsáki and Chrobak, 1995; Rinzel et al., 1998).

Here we examine a network of inhibitory interneurons in the

\footnotetext{
Received Oct. 9, 1998; revised Dec. 30, 1998; accepted Feb. 10, 1999.

This work was supported by The Israel Science Foundation and the European Commission.

Correspondence should be addressed to Y. Yarom, Department of Neurobiology, Hebrew University, Givat Ram, 91904, Jerusalem, Israel.

Copyright (C) 1999 Society for Neuroscience 0270-6474/99/193298-09\$05.00/0
}

mammalian cerebellar cortex whose structure has been described in great detail (Palay and Chan-Palay, 1974). There are three main types of inhibitory GABAergic interneurons: Golgi cells in the granular layer and the interneurons in the molecular layer, the basket and stellate cells. The mutually connected molecular layer interneurons receive excitatory input from parallel and climbing fibers and innervate the principle neurons of the cerebellar cortex, the Purkinje cells. Basket and stellate cells have relatively short dendritic trees, which are organized in an almost planar structure, and their axons always extend perpendicular to the parallel fibers. Whereas the axons of basket cells create a basketlike structure around the Purkinje cell somata, stellate axons terminate on the dendritic trees of the Purkinje cells. The axons of stellate cells are either confined to the proximal area of their cell bodies $(30-40 \mu \mathrm{m})$ or extend up to $450 \mu \mathrm{m}$ from their cell bodies (Palay and Chan-Palay, 1974; Sultan and Bower, 1998).

Structural considerations suggest two possible roles for the molecular layer interneurons. First, because of their joint source of excitation, the local axons of stellate cells can provide each Purkinje cell with inhibition, which is proportional to the amount of its excitation (Marr, 1969; De Schutter and Bower, 1994). Second, the length and orientation of the longer axons of the molecular layer interneurons enables them to induce lateral inhibition (Eccles et al., 1967). By recording simultaneously from pairs of inhibitory neurons in the molecular layer, we show here that these interneurons are organized in local networks, interconnected by weak electrical synapses. These networks synchronize their activity over a wide time window.

\section{MATERIALS AND METHODS}

Slice preparation. Sagittal slices (300- $\mu \mathrm{m}$-thick) were prepared from the vermis of a guinea pig cerebellum. Guinea pigs $(80-120 \mathrm{gm})$ were 
anesthetized intraperitonally with pentobarbitone $60 \mathrm{mg} / \mathrm{kg}$ and perfused through the heart with $100 \mathrm{ml}$ of cold $\left(0-1^{\circ} \mathrm{C}\right)$ oxygenated physiological solution (containing in mM: $124 \mathrm{NaCl}, 5 \mathrm{KCl}, 1.3 \mathrm{MgSO}_{4}, 1.2$ $\mathrm{KH}_{2} \mathrm{PO}_{4}, 26 \mathrm{NaHCO}_{3}, 10$ glucose, and $2.4 \mathrm{CaCl}_{2}, \mathrm{pH} 7.4$, aerated with $95 \% \mathrm{O}_{2}$ and $5 \% \mathrm{CO}_{2}$ ). After decapitation, the cerebellum was quickly removed and sliced (Campden Instruments LTD 752M vibroslice) in cold sucrose solution (containing in mM: $5 \mathrm{KCl}, 1.3 \mathrm{MgSO}_{4}, 1.2$ $\mathrm{KH}_{2} \mathrm{PO}_{4}, 26 \mathrm{NaHCO}_{3}, 10$ glucose, $2.4 \mathrm{CaCl}_{2}$, and 124 sucrose). The slices were incubated in the sucrose solution at room temperature for 20 min, after which the sucrose solution was slowly replaced by normal physiological solution over a period of $1 \mathrm{hr}$. This procedure was found to be crucial for the viability of the stellate and basket cells. Slices were kept at room temperature in an oxygenated physiological solution until they were transferred into the recording chamber.

Recordings. The recording chamber, mounted on an upright microscope stage (Zeiss Axioskop), maintained a constant temperature of $30^{\circ} \mathrm{C}$ by a temperature control unit and was continuously perfused with aerated physiological solution. Drugs were added to the physiological solution to reach final concentrations of: TTX, $0.1 \mu \mathrm{M}$ (Molecular Probes, Eugene, OR); CNQX, $50 \mu \mathrm{M}$ (Research Biochemicals, Natick, MA); bicuculline, $50 \mu \mathrm{M}$ (Sigma, St. Louis, MO). Low Ca ${ }^{2+}$ solution contained $0.5 \mathrm{mM} \mathrm{Ca}^{2+}$ and $10 \mathrm{~mm} \mathrm{Mg}^{2+}$. Slices were perfused for 10 min with the solution containing the drug before the examination of its effect. The molecular layer interneurons in the slice were readily identified using infrared differential interference contrast optics, and wholecell patch recordings were quickly attained. The patch pipettes were pulled on a Narishige pp-83 puller and had a DC resistance of 10-12 $\mathrm{M} \Omega$. In intracellular recordings the pipette solution contained (in $\mathrm{mM}$ ): $140 \mathrm{~K}$-gluconate, $4 \mathrm{NaCl}, 0.5 \mathrm{CaCl}_{2}, 5 \mathrm{EGTA}, 3 \mathrm{Mg}$-ATP, and 10 HEPES, pH 7.2. When QX-314 was used, it was added to the pipette solution in concentration of 0.5 or $10 \mathrm{~mm}$. Recordings were made with an Axoclamp 2B (Axon instruments, Foster City, CA) in current-clamp mode. Two identical amplifiers were used for simultaneous recordings from two cells. Extracellular recordings were performed with the same amplifiers. The patch pipette, which was placed near the cell membrane without generating a seal, was filled with extracellular solution. Data were stored on video cassette (Neurocorder DR-484) for off-line analysis.

Morphological procedure. Biocytin (0.5-1\%, Sigma) was injected intracellularly using short hyperpolarizing pulses of $4 \mathrm{msec}, 50-100 \mathrm{pA}$ at 20 $\mathrm{Hz}$ for $2 \mathrm{~min}$. After $1 \mathrm{hr}$ of incubation at $30^{\circ} \mathrm{C}$, the slice was fixed overnight at $4^{\circ} \mathrm{C}$ in $2 \%$ paraformaldehyde, $0.2 \%$ picric acid, and $0.1 \%$ glutaraldehyde in $0.1 \mathrm{M}$ cacodilate buffer. After rinsing several times with phosphate buffer, slices were treated with $0.5 \%$ sodium borohydride to prevent nonspecific staining, then rinsed and treated with $\mathrm{MeOH}(10 \%)$ and $\mathrm{H}_{2} \mathrm{O}_{2}(3 \%)$ to block endogenous peroxidases. The slices were then incubated for at least $3 \mathrm{hr}$ in biotinylated horseradish peroxidase conjugated to avidin (ABC kit; Vector Laboratories, Burlingame, CA) with Triton X-100 (0.5\%), then rinsed and developed under visual control using the DAB reaction.

Analysis. To analyze the coupling between neurons, we assume that coupling can be modeled as two point neurons with input resistance $R 1$ and $R 2$, coupled by a resistance $R c$. Current pulse $(I)$ injected into cell 1 will generate a voltage response in cell $1(V 1)$ as well as in cell 2 (V2). The coupling coefficient (CC) defined as $V 2 / V 1$ is equal to:

$$
\mathrm{CC}=1 /(1+R \mathrm{c} / R 2)
$$

The coupling coefficient was experimentally measured from the average voltage responses to hyperpolarizing or depolarizing pulses of various intensities within the range in which $R 2$ is constant. Current was injected in turn into each cell of a pair allowing two coupling coefficients to be calculated for each pair.

A software program written in Labview was used to calculate the spike cross-correlogram. Data were sampled at $10 \mathrm{kHz}$, and spikes were detected by threshold peak detector. In cases of low signal-to-noise ratio (as in extracellular measurements), sampling rate was increased to $20 \mathrm{kHz}$, and a peak was considered valid only if at least four consecutive points were above the predetermined threshold. This excluded high-frequency noise from being counted as spikes. The spike cross-correlogram was calculated for 50-100 traces of $1 \mathrm{sec}$ duration, with $1 \mathrm{msec}$ time bins. Each histogram was normalized for firing rate and number of traces by dividing the number of observed spikes by the expected number of spikes, assuming a random firing of two neurons. The expected number of spikes, $E(t)$, was calculated using Equation 2:

$$
\mathrm{E}(t)=\lambda_{1} * \lambda_{2} * \Delta t * N *(T-|t|)
$$

Where $\lambda_{1}$ and $\lambda_{2}$ are the firing rates of cells 1 and 2, respectively, $\Delta \mathrm{t}$ the bin size (1 msec), $N$ the number of traces, and $T$ the trace duration (1 $\mathrm{sec})$. The normalized histogram thus expresses the deviation of the results from randomly occurring spikes. The synchronization width was defined as the width of the cross-correlogram at the $99 \%$ confidence limit. The synchronization strength was defined as the integral of the normalized histogram over the synchronization width.

\section{RESULTS}

The spontaneous activity of molecular layer interneurons in the slice preparation has been well documented (Llano and Gerschenfeld, 1993; Häusser and Clark, 1997; Kondo and Marty, 1998). In our preparations, the spontaneous firing rate recorded extracellularly ranged between 4 and $34 \mathrm{~Hz}$ with an average of $13 \pm 9 \mathrm{~Hz}(n=21)$. Autocorrelograms of the spontaneous activity of these cells revealed regular rhythmic activity in 13 cells, whereas the rest of the cells fired irregularly. Because the spontaneous activity depends on the membrane potential, which may be affected by intracellular recordings, a negative DC current was usually injected into the cell to maintain a membrane potential of -45 to $-55 \mathrm{mV}$. At this membrane potential, the rate of spontaneous activity was in the same range as that measured extracellularly. These results fit those from previous studies (Häusser and Clark, 1997).

Simultaneous recordings from pairs of adjacent molecular layer interneurons revealed a significant tendency for synchronized firing in $40 \%$ of the recordings $(n=58)$, which can frequently be seen in visual inspection of both extracellular (Fig. $1 A$ ) or intracellular (Fig. $1 C$ ) recordings. To quantify this observation, the normalized cross correlation histogram of the spike trains was calculated (see Materials and Methods) and plotted in Figure 1, $B$ and $D$, for the extracellular and intracellular recordings, respectively. The clear peak around time 0 indicates the strong tendency of these two pairs to fire in synchrony, with a synchronization strength and width (see Materials and Methods) of 10.4 and 13 msec, respectively, for the extracellular recordings and 2.68 and $11 \mathrm{msec}$ for the intracellular recordings. The synchronization strength in all the recordings ranged between 1.52 and 10.96 , with an average of $3.6 \pm 2.5$. The mean synchronization width was $12.8 \pm 4 \mathrm{msec}(n=22)$. It should be noted that the distribution around time 0 was not symmetrical in about half the pairs, particularly with extracellular recordings.

Synchronized firing may be caused by chemical synaptic transmission, electrical synapses, or nonsynaptic interactions. To examine the possibility that chemical synapses are involved in the synchronized firing, we used bicuculline, CNQX, or low $\mathrm{Ca}^{2+}$ solution to block the inhibitory, excitatory, or all synaptic connections, respectively. Bicuculline $(50 \mu \mathrm{M})$ had no significant effect on the cross-correlogram (Fig. 1E). The synchronization strength and duration in this pair of neurons were 10.96 and 12 msec, respectively, in control conditions, and 10.63 and $11 \mathrm{msec}$ in the presence of bicuculline. Similar results were observed in four additional neuron pairs. It should be mentioned that blocking the inhibitory synapses occasionally increased the regularity of the spontaneous activity (Häusser and Clark, 1997). Blocking the excitatory synapses similarly failed to effect the synchronicity in two pairs of neurons in which the effect of CNQX $(50 \mu \mathrm{M})$ was examined (Fig. $1 F$ ). Finally, lowering the $\mathrm{Ca}^{2+}$ concentration in the external solution, thereby completely blocking all chemical synaptic interactions, did not effect the synchronization as well (results not shown). We therefore conclude that chemical synaptic transmission is not involved in the synchronization of firing.

The possible involvement of electrical synapses in the synchro- 
A

Cell 1

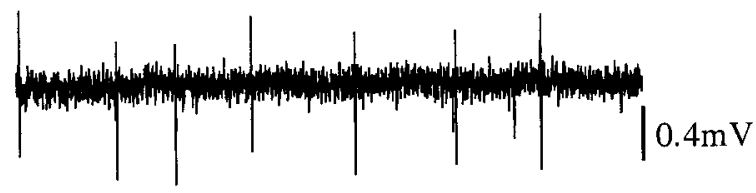

Cell 2

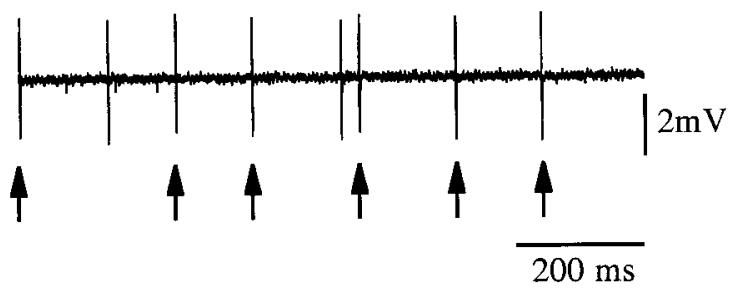

Cell 1

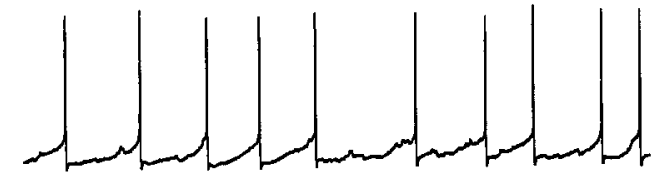

Cell 2

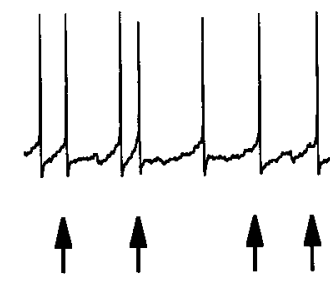

E

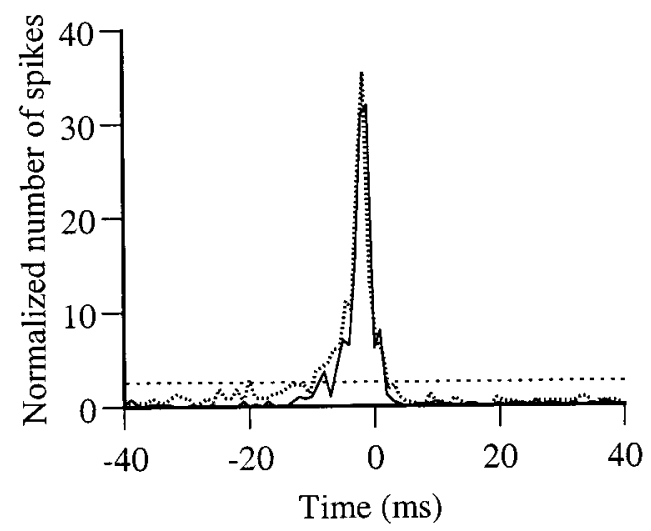

B

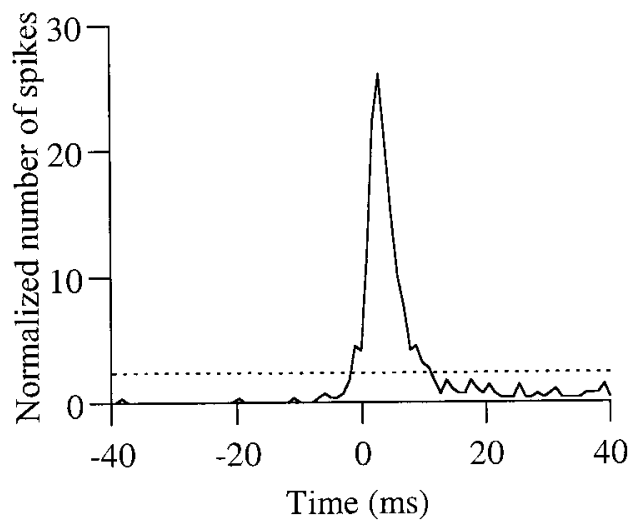

D

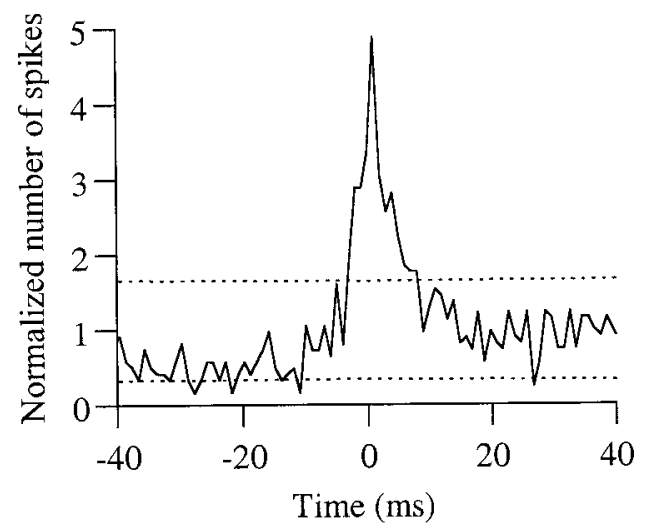

F

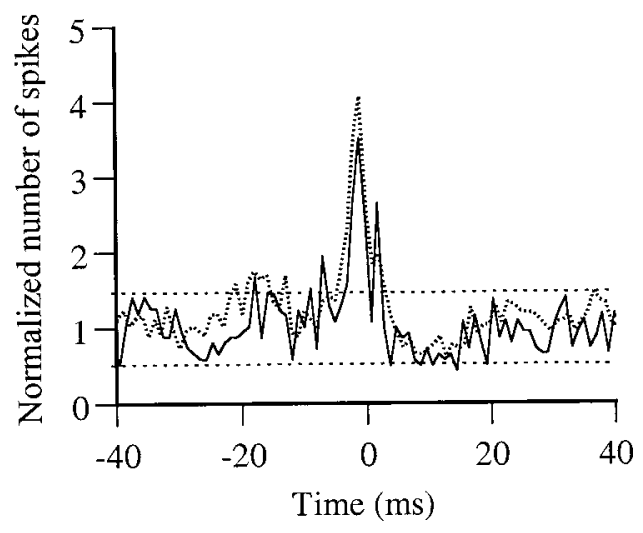

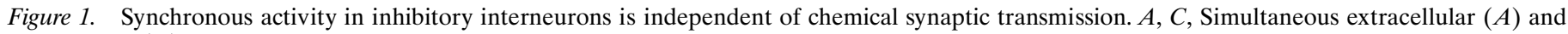

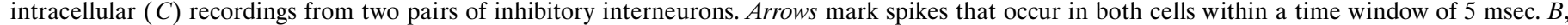

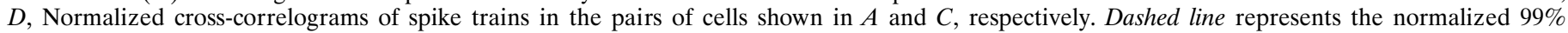

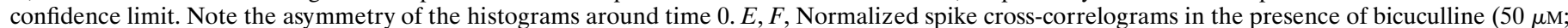
$E)$ and CNQX $(50 \mu \mathrm{M} ; F)$. Dashed and continuous plots represent the control condition and the presence of the blocker, respectively.

nized firing was examined using simultaneous intracellular recordings from two adjacent neurons (Fig. 2). Current pulses of various intensities were injected intracellularly into cell 1 (Fig. $2 A$, bottom traces), and the voltage responses were observed in both cells (Fig. $2 A$, top and middle traces). The responses in cell
2 , however, were an order of magnitude smaller than those in cell 1. These results, which clearly demonstrate direct current flow between neurons, were obtained in $40 \%$ of the recordings $(n=$ 38 ). In 30 pairs of neurons, in which cross-correlogram was calculated and direct current flow was examined, nine pairs 


\section{A B}

Cell 1 - Voltage

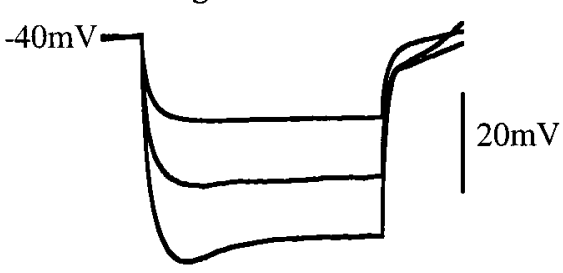

Cell 2 - Voltage

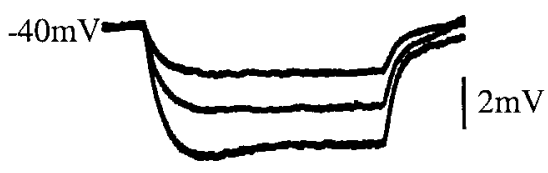

Cell 2 - Voltage

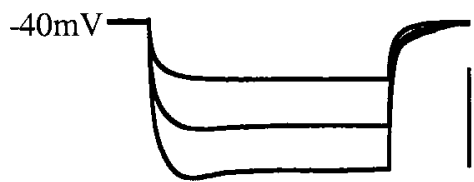

Cell 1 - Injected current

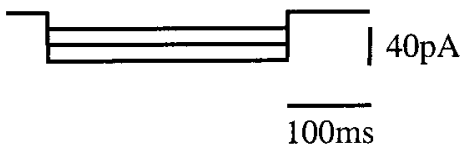

Cell 2 - Injected current

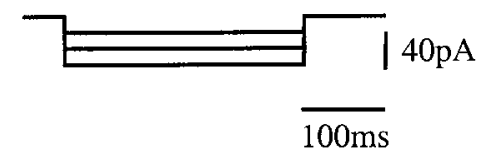

$20 \mathrm{mV}$

C

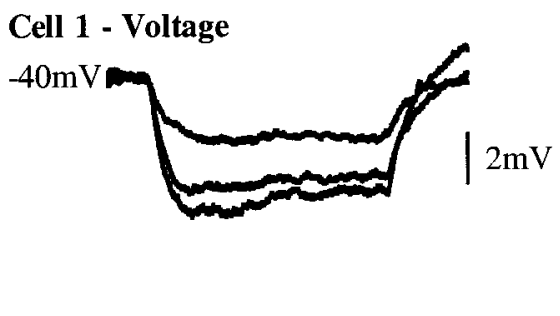

Postsynaptic
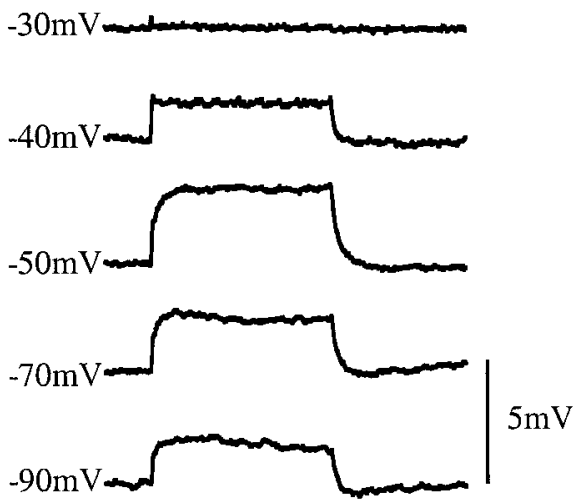

\section{Presynaptic}

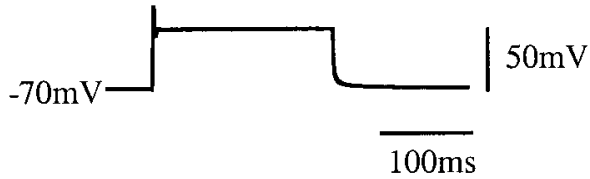

D

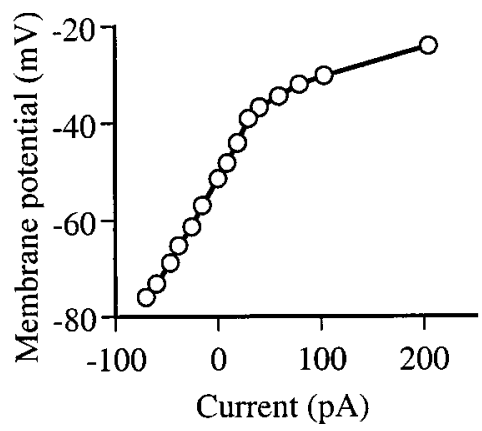

$\mathbf{E}$

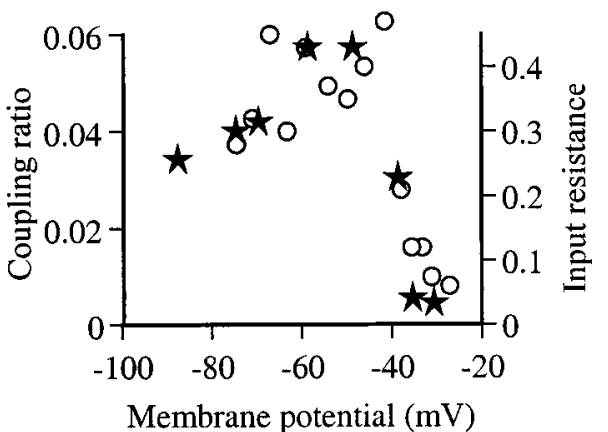

F

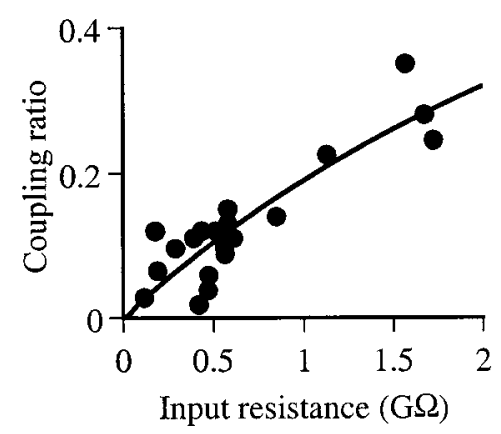

Figure 2. Simultaneous recordings from two neurons reveal direct current flow between two inhibitory interneurons. $A$, Pulses of current of different amplitudes were injected to cell 1 (bottom traces). Voltage responses were measured in cell 1 (top traces) and in cell 2 (middle traces). Note the difference in scale. $B$, Current was injected to cell 2 (bottom traces), and voltage responses were recorded in both cells (top and middle traces). $C$, The postsynaptic response is voltage-dependent. Current pulses of constant amplitude were injected into the presynaptic cell (voltage responses in presynaptic cell, bottom trace), and the voltage response in the postsynaptic cell was measured at different membrane potentials, set by DC current injection. Note the reduction in coupling at depolarizing membrane potentials. $D$, The current-voltage curve of the postsynaptic cell in $C$. Note the large and abrupt rectification at potentials above $-38 \mathrm{mV}$ and the small anomalous rectification at potential below $-70 \mathrm{mV}$. E, The input resistance (circles) and coupling coefficient (stars) of the cell shown in $C$, as a function of membrane potential. $F$, The coupling coefficient between 21 pairs of inhibitory interneurons as a function of the input resistance of the postsynaptic cell. The line is the curve of best fit plotted according to equation 1. Coupling resistance was calculated to be $4.25 \mathrm{G} \Omega$, and $R^{2}$ was 0.77 . All the above experiments were performed in the presence of $0.1 \mu \mathrm{M}$ TTX.

showed both synchronized firing and direct current flow. The rest of the neuron pairs (21) showed neither direct current flow nor synchronization. The coupling coefficient (see Materials and Methods) for the pair of cells shown in Figure $2 A$ was 0.124 . The coupling coefficient over the whole sample under resting conditions ranged between 0.018 and 0.28 with a mean of $0.12 \pm 0.07$ $(n=24)$.

The results of the complementary experiment, namely current injection into cell 2, are shown in Figure $2 \mathrm{~B}$. In this case, the coupling coefficient was 0.185 , almost $50 \%$ larger than when current was injected into cell 1 (Fig. $2 A$ ). Nonsymmetrical current flow was found in 6 of 12 pairs of neurons. The ratio of the two coupling coefficients for these six pairs of neurons ranged between 1.85 and 8.04, with an average of 3.26 (ratios between pairs of coupling coefficients of up to 1.25 were considered symmetrical, and were not included in the average). It is reasonable to assume that this asymmetrical current flow contributes to the asymmetrical distribution of the cross-correlograms around time 0 . However, we could not find any correlation between the asymmetric current flow and the asymmetry of the cross-correlograms. 
Thus, it is likely that there are additional factors that contribute to the asymmetry of the cross-correlograms. For example, in some cases depolarizing the membrane potential of one cell can modulate the cross-correlogram, probably by holding the membrane potential closer to the threshold, thus making this cell more responsive to any depolarizing input. This might be the reason why asymmetric cross-correlograms were more often found with extracellular recordings where the resting potential could not be modulated by current injection, as in the case of intracellular recordings.

Asymmetric coupling between neurons results either from a rectifying coupling conductance or from differences in the input resistance of the cells, or both. The input resistance of the molecular layer interneurons depends on their membrane potential. We therefore tested the effect of the input resistance on the coupling strength by manipulating the membrane potential. Figure $2 D$ shows a typical current-voltage relationship of a molecular layer interneuron in the presence of TTX $(0.1 \mu \mathrm{M})$. The significant and abrupt rectification, which occurs at a membrane potential of $-35 \mathrm{mV}$, reflected as a 10 -fold decrease in the input resistance. As expected, the coupling between two cells also showed a similar dependence on membrane potential. Figure $2 C$ shows that the voltage response of a postsynaptic cell (top traces) to a constant voltage change in the presynaptic cell (bottom trace) depends on the postsynaptic membrane potential. Maximum responses were obtained at a membrane potential of $-50 \mathrm{mV}$. A somewhat smaller response was recorded at hyperpolarizing membrane potentials $(-90 \mathrm{mV})$, and the responses decreased greatly and almost disappeared when the membrane was depolarized to $-30 \mathrm{mV}$. A comparison between the dependence of the input resistance (circles) and the coupling coefficient (stars) on the postsynaptic membrane potential is shown in Figure $2 E$. The similarity between the two shows that the input resistance of the postsynaptic cell modulates the coupling coefficient.

The relationship between the input resistances of 21 postsynaptic cells and their coupling coefficients is plotted in Figure $2 F$. As is to be expected, the higher the input resistance, the larger the coupling coefficient. The predicted relation between these two parameters is given in Equation 1 (see Materials and Methods). The curve (Fig. $2 F$, line) that best fits our results yields a coupling resistance $(R c)$ of $4.25 \mathrm{G} \Omega$ and has $\mathrm{R}^{2}$ of 0.77 . The significant fit of the results to the predicted curve has several implications. First, differences in the coupling coefficient can be attributed to differences in input resistance and, therefore, the asymmetrical current flow between two cells is most likely caused by differences in the input resistance of these cells. Second, the good fit of the theoretical curve to the experimental results suggests similar coupling resistances between the various pairs of neurons. This is a rather surprising result because the coupling resistance reflects the number of gap junctions, as well as their location relative to the cell body.

Having demonstrated direct current flow between neurons, we then proceeded to examine its significance, particularly whether the current generated by the brief action potential is sufficient to induce synchronized firing. To answer this question, we analyzed the postsynaptic potential during spontaneous firing of the presynaptic cell. Figure $3 A$ shows the average of only the subthreshold voltage response of cell 2 (top trace) aligned by a spike in cell 1 (bottom trace). This response had two components: a prolonged depolarizing potential and a short, attenuated spikelet (half width $2.4 \mathrm{msec}$; amplitude, $1.15 \mathrm{mV}$ ), which coincided with the action potential in cell 1 . As would be expected, the coupling coefficient for the brief spike (average, $0.025 \pm 0.007 ; n=12$ ) was smaller than that calculated for long current pulses.

The prolonged depolarizing component is particularly interesting; presumably it reflects the slow depolarizing process leading to the presynaptic action potential. However, it continues beyond the duration of the spike. A possible explanation is that voltagedependent currents in the postsynaptic cell contribute to this slow depolarizing process. This was examined by hyperpolarizing the postsynaptic cell to prevent activation of voltage-dependent currents. Figure $3 B$ indeed shows that, under these conditions, the prolonged depolarization in the postsynaptic cell was reduced.

The three superimposed traces in Figure $3 C$ show the postsynaptic responses at the two membrane potentials (Fig. $3 A, B$, top traces) and their relation to the presynaptic action potential (Fig. $3 B$, bottom trace). Up to the point marked by the arrow, the two postsynaptic responses follow the same trajectory. Beyond this point, the postsynaptic response at the more depolarized potential had a steeper slope, and it proceeded beyond the duration of the spikelet. In contrast, at the more hyperpolarized potential, the membrane voltage returns to baseline after the spikelet. In both cases, the peak of the spikelets coincides with the falling phase of the presynaptic action potential.

The contribution of postsynaptic currents to the compound response was further assessed by blocking postsynaptic sodium channels with intracellular QX-314 (0.5 mM). Under this condition, the postsynaptic action potentials were blocked almost immediately after breaking the neuron membrane and establishing the whole-cell recording. Figure $3 D-F$ displays the results of a QX-314 experiment in the same format as that shown in Figure $3 A-C$. The postsynaptic response in cell 2 (the cell injected with QX-314) shows only a spikelet, whereas the prolonged depolarizing component is absent. Furthermore, depolarizing the postsynaptic cell to $-44 \mathrm{mV}$ failed to recover the prolonged depolarization. We conclude that the voltage dependence of the postsynaptic response is caused by postsynaptic, voltagedependent, QX-314-sensitive currents. Because, under normal conditions, the membrane potential lies within the voltage range where these currents are active, the most common subthreshold postsynaptic response will be similar to that shown in Figure $3 A$. Interestingly, the action potentials in the presynaptic cell were unaffected even after $1 \mathrm{hr}$ of recordings, using $10 \mathrm{~mm}$ intracellular QX-314. This suggests that the coupling path prevented the diffusion of the QX-314 to the coupled cell.

The cross-correlogram of the spike trains in these cells is shown in Figure $4 A$. The synchronization width was $12 \mathrm{msec}$, which is much broader than the time-to-peak of the spikelet ( 1 msec), as measured in Figure $3 A$. To further assess the timing between the firing of the neurons, we aligned 50 successive cell 2 traces by a spike in cell 1 (Fig. $4 B$ ). Of the 17 spikes that occurred within the time window of significant correlation after the presynaptic spike, only five spikes occurred within $<1 \mathrm{msec}$. Thus, it appears that only $30 \%$ of the synchronized spikes were actually elicited by the spikelets. Similar results were obtained when traces of cell 1 were aligned by spikes in cell 2 (Fig. $4 C$ ).

The functional significance of this coupling depends on its prevalence and spatial organization. We measured the extent of dye coupling by adding biocytin $(1 \%)$ to the pipette solution. Dye coupling was found in $47 \%$ of dye injections to single cells, spreading to up to nine neighboring cells, including stellate as well as basket cells. However, the distribution of dye coupling was nonuniform. Dye coupling occurred more frequently, and larger groups of cells were stained, when dye was injected to superficial 

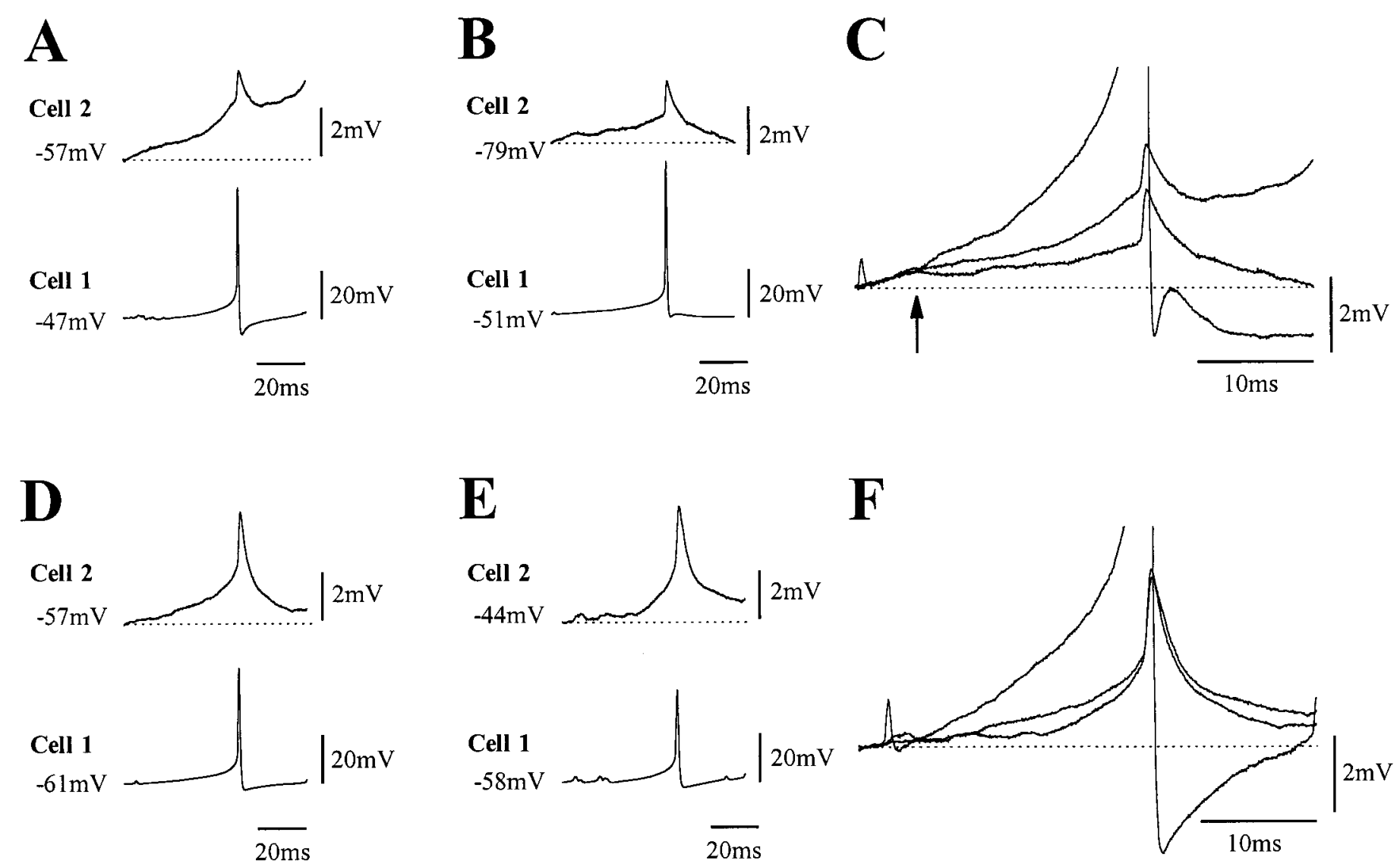

Figure 3. Intrinsic currents amplify the coupling. $A$, Spike-triggered average of the subthreshold response in cell 2 to a spike in cell 1 . Traces were selected according to the criteria that cell 2 did not respond with an action potential before and at least 20 msec after the spike in cell 1 . The horizontal dashed line represents the average resting membrane potential of cell 2 as measured $40 \mathrm{msec}$ before the action potential in cell 1 . Note the prolonged depolarization and the spikelet in cell 2 that characterizes the postsynaptic response. $B$, The subthreshold response measured as in $A$ during hyperpolarization of cell 2 to $-79 \mathrm{mV}$ by DC current injection. Under these conditions the prolonged depolarizing response is attenuated, and the membrane potential declines to resting level after the spikelet. Note that resting potential in cell 1 was also affected, increasing the amplitude of the action potential in cell 1 and the spikelet in cell 2. $C$, Three superimposed traces of the action potential in cell 1 and the spikelets in cell 2 under normal and hyperpolarizing conditions. $D-F$, Same as $A-C$, cell 2 injected with $0.5 \mathrm{~mm}$ QX-314. Note the independence of the postsynaptic response on the membrane potential and the absence of the postspikelet depolarizing response $(F)$, indicating its dependence on QX-314-sensitive currents.

stellate cells as compared with deep stellate and basket cells (Table 1). As shown in Figure $5 A-C$, the stained cells within a group tended to lie in the same sagittal plane. Each of the two superficial groups shown in Figure 5, $A$ and $C$, contain five stained neurons. Note that their intermingled dendrites occupy a limited area of $\sim 100 \times 100 \mu \mathrm{m}$. The two stained axons in Figure $5 C$ spread over $250 \mu \mathrm{m}$ in the sagittal plain. Figure $5 B$ shows an example of another type of group, one composed of a basket cell, identified by its characteristic axonal ramifications, and a stellate cell (arrow).

Taking together all these observations, it is reasonable to conclude that the inhibitory interneurons of the cerebellar cortex are organized in specific networks that communicate via electrotonic coupling.

\section{DISCUSSION}

The main and novel finding of this in vitro investigation is that $40 \%$ of the simultaneously recorded interneurons in the molecular layer of the cerebellar cortex are electrically coupled. This coupling is strong enough to generate synchronized firing. Intracellular staining revealed groups of indirectly stained neurons, therefore it is reasonable to assume that these interneurons form local networks that give rise to synchronized activity.

Our physiological and morphological experiments were performed on cerebellar brain slices, thus we must eliminate the possibility that this electrical coupling is an experimental artifact of the in vitro preparation. Although incidents of fusion of distal dendrites caused by the preparation procedure have been reported (Gutnick et al., 1985), there are several arguments against this possibility. First, the coupling resistance derived from our results is in the order of several gigaohms (Fig. 2), much larger than expected from the axial resistance of the dendritic tree. Crude calculation shows that a dendritic fusion located $100 \mu \mathrm{m}$ from the cell body on a $1 \mu \mathrm{m}$ diameter dendrite will result in a coupling resistance of only $100 \mathrm{M} \Omega$. Second, the almost twodimensional structure of these neurons minimizes the possibility of dendritic damage during slice preparation. Furthermore, the probability of encountering electrically coupled or dye-coupled cells was independent of the cells depth within the slice. Third, adding QX-314 intracellularly to one cell failed to affect the action potentials in the coupled cell. Action potentials in the injected cell were readily blocked, even in a concentration as low 


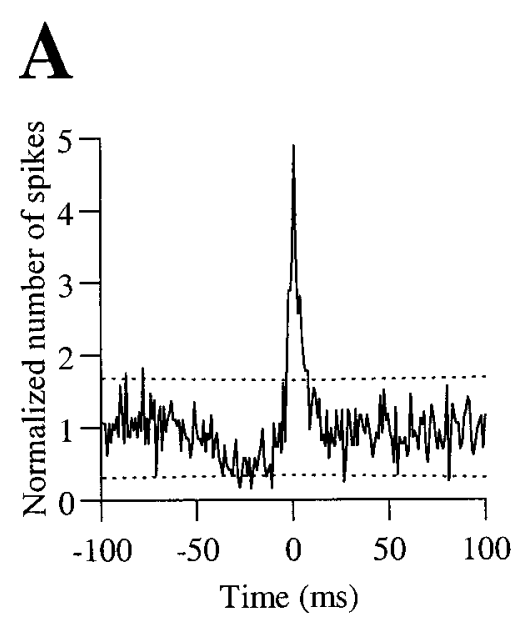

B

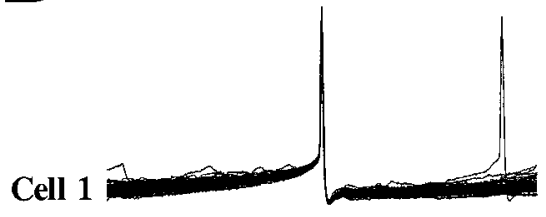

Cell 1

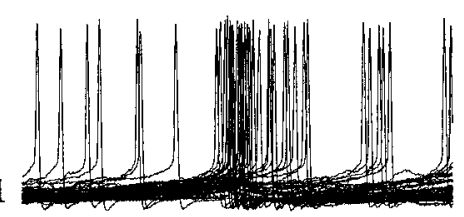

Cell 2

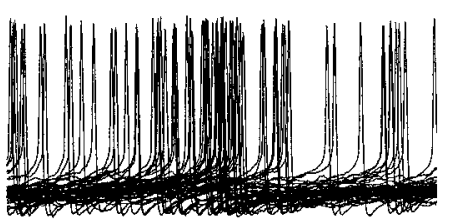

$20 \mathrm{mV}$

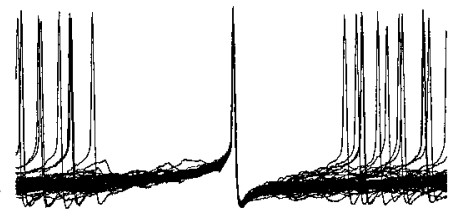

Figure 4. The interaction between current flow and intrinsic currents increases the time window of synchronization. All three panels were measured from the same neuron pair. $A$, Normalized spike cross-correlogram of spontaneous activity in the two cells. $B$, Fifty consecutive spontaneous activity traces of cell 1 (top traces) and cell 2 (bottom traces) aligned by a spike in cell 1 . Note the wide and asymmetric distribution of the spikes in cell 2 around the time of the spike in cell 1 . $C$, Same as $B$, but traces were aligned according to the spike in cell 2 .

Table 1. Prevalence of dye coupling and the size of stained groups in different depths of the molecular layer

\begin{tabular}{llll} 
& $\begin{array}{l}\text { Inner third } \\
(N=10)\end{array}$ & $\begin{array}{l}\text { Middle third } \\
(N=8)\end{array}$ & $\begin{array}{l}\text { Outer third } \\
(N=14)\end{array}$ \\
\hline $\begin{array}{l}\text { Percentage of multiple staining } \\
\text { Number of stained cells in a }\end{array}$ & $20 \%$ & $37 \%$ & $71 \%$ \\
$\quad \begin{array}{l}\text { group } \\
\text { Range } \\
\text { Average }\end{array}$ & 2 & $2-4$ & $2-9$ \\
\hline
\end{tabular}

as $0.5 \mathrm{~mm}$. Because the action potentials in the coupled cell were not affected even after $1 \mathrm{hr}$ using a $10 \mathrm{~mm}$ concentration of the drug, we are bound to conclude that QX-314 did not reach the coupled cell. Similar results were described by Logan et al. (1996) when the QX-314, which was intracellularly injected into sympathetic preganglionic neurons, failed to effect the presynaptic action potentials. It is reasonable to assume that QX-314 is impermeable through gap junction, and therefore this observation argues against dendritic fusion and supports the possibility of an electrotonic junction. Finally, ultrastructural studies by Sotelo and Llinás (1972) have demonstrated gap junction structures between perikarya and dendrites of the inhibitory interneurons in the molecular layer. In view of these arguments, we think it justified to conclude that the electrotonic coupling seen in our preparation is not an experimental artifact.

At this point one should mention that Vincent and Marty (1996), in their study of molecular layer interneurons, failed to find synchronized activity or dye coupling in these neurons. The source for this discrepancy could lie in their use of 9- to 15-d-old rats, as opposed to our mature guinea pigs, in which the dendrites of superficial stellate cells and the electrical connections may still be in the process of development (Ito, 1984) (postnatal development of gap junctions has been reported by Bourrat and Sotelo, 1983). A preference to record from basket cells by Vincent and Marty (1996) is also a plausible explanation. At this early developmental stage the big basket cells are more likely to be selected for recordings. This would be in agreement with our experiments where basket cells were rarely connected.
In characterizing the electrical coupling between the molecular layer interneurons, we estimate the coupling resistance between neurons to be of the order of gigaohms. This estimate, calculated from the relationship between the coupling coefficient and the input resistance of the postsynaptic cell, is based on several assumptions. First, the coupled neurons are modeled as single compartments, meaning that the axial resistance from cell bodies to the site of the gap junction is lumped into the coupling resistance. However, as mentioned, the axial resistance of the dendritic tree is of the order of $100 \mathrm{M} \Omega$. Even if the length was increased to $200 \mu \mathrm{m}$, it would still be an order of magnitude smaller than the estimated coupling resistance. Second, we have assumed that the measured input resistance represents the true value of the input resistance but, in fact, it is an underestimation of the actual value. Only part of the injected current used to calculate resistance flowed through the input resistance of the cell, whereas the remainder flowed through the coupling resistance and the input resistance of the coupled cell. Thus, calculating the input resistance as the ratio between the voltage response and the injected current underestimates the input resistance. Accordingly, an order of magnitude difference between the input resistance and the coupling resistance leads to a $10 \%$ underestimation of the input resistance. This underestimation reduces the estimation of the coupling resistance by about the same percentage. Third, in fitting the theoretical curve to the experimental results we assumed that the coupling resistance is similar for all recorded pairs, as indicated by our results. To further check this point, we calculated the same curve for a case in which the coupling coefficient between a pair of neurons was measured, whereas the input resistance of the postsynaptic cell was set to different values (Fig. $2 C$ ). This gave a coupling resistance of $6.8 \mathrm{G} \Omega$ with a correlation factor of 0.94 . Thus, it is reasonable to conclude that (1) the coupling resistance between the molecular layer interneurons is in the order of $5 \mathrm{G} \Omega$ with relatively small variance, (2) that the added coupling resistance caused by the location of the coupling site along the dendritic tree is negligible, and (3) that the asymmetric coupling is caused by differences in input resistance of the postsynaptic cell.

The electrotonic coupling depends on the voltage of the postsynaptic cell, and this voltage dependence is expressed in two 


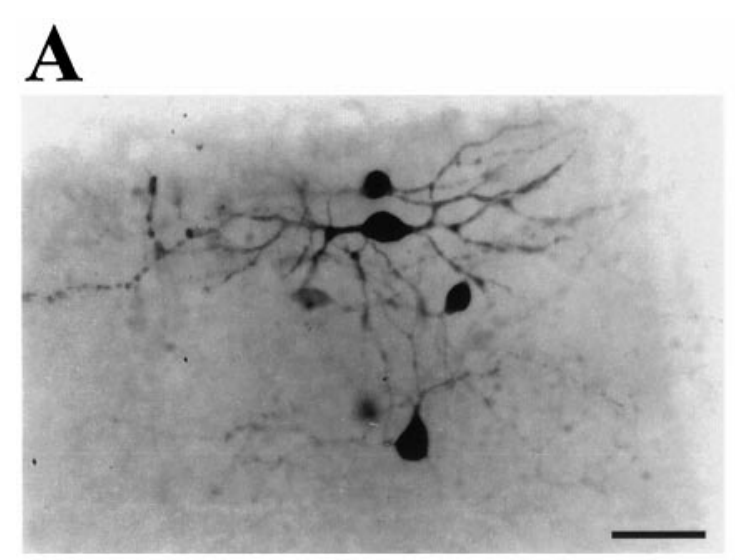

B

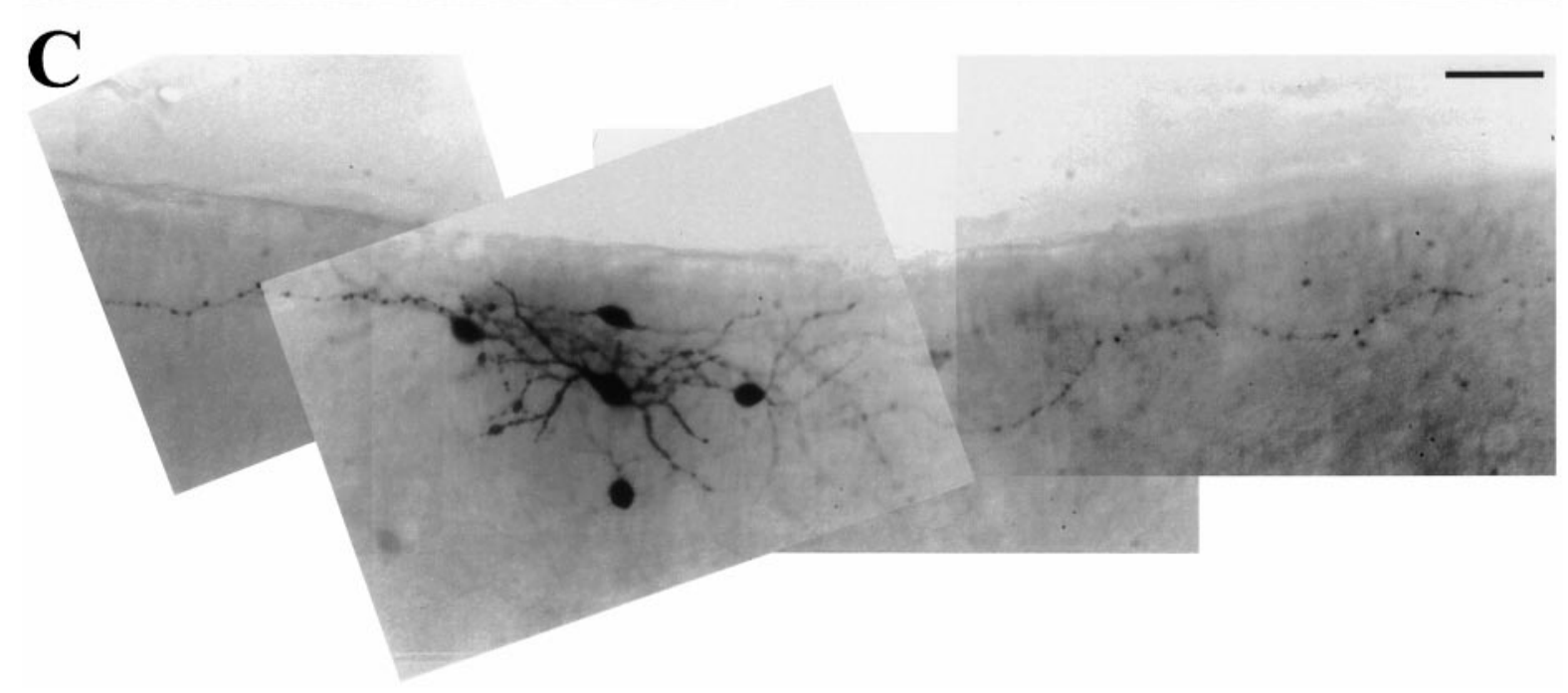

Figure 5. Dye coupling between molecular layer inhibitory interneurons. Only a single neuron was injected with biocytin in each example. $A$, A superficial group of dye-coupled neurons. Typically the cell bodies are in close proximity, and the dendrites intermingle. $B$, A deep group of dye-coupled neurons composed of a basket cell and a single stellate cell (arrow). Note the typical structure of the axon and the elaborated dendritic tree of the basket cell. The outlines of the cell bodies of nearby unstained Purkinje cells are drawn for clarification. $C$, A superficial group of coupled stellate cells showing the sagittal organization of their axons. Scale bars, $20 \mu \mathrm{m}$.

ways: a modulation of coupling strength caused by the voltage dependence of the input resistance (Fig. 2C) and an increase in apparent coupling by intrinsic currents that amplify the postsynaptic responses (Fig. 3). This latter is particularly interesting because it contributes significantly to what we regard as the physiological role of the coupling. Specifically, a weakly coupled network of spontaneously active neurons can generate synchronized firing within a time window that is larger than would be predicted from the time course of the presynaptic action potential. This discrepancy results from intrinsic currents that operate between the resting and the threshold potentials, generating a slow depolarization that determines the spontaneous activity of the neurons. The slow depolarization propagates via the gap junctions to activate the same type of currents in the postsynaptic cell. The slow process of depolarization proceeds in both cells until threshold is reached and an action potential is elicited in one of them. The current generated by the action potential also propagates via the gap junctions and, if the combined depolarization of the postsynaptic cell reaches threshold, the cell will fire within a short time window of a few milliseconds. If the spikelet fails to activate the postsynaptic cell, the slow depolarizing process continues until it eventually leads to a postsynaptic action potential. Thus, although the postsynaptic action potential is synchronized with the presynaptic action potential, the time window will be relatively wide. This was exactly what was seen here; the width of the cross-correlogram was wider than would be predicted from the rise time of the spikelet.

The time window of synchronization may also be increased if the spikelet sums with spontaneous synaptic activity. However, as shown in Figure 1, the width of the cross-correlogram does not change when synaptic blockers are applied. It therefore appears that it is these intrinsic currents that amplify the coupling coefficient and widen the time window of synchronization.

At this stage, one can only speculate on the physiological relevance of synchronized activity in the cerebellar inhibitory interneurons. Two points appear to be functionally significant: the spatial distribution of the axons of the interneurons and the size difference between the superficial and the deep local networks. The superficial neurons appear to innervate the distal dendrites of the Purkinje cells. Callaway et al. (1995) have demonstrated that the superficial neurons are very effective at blocking the $\mathrm{Ca}^{2+}$ responses evoked by the climbing fibers. It has been suggested that their role is to prevent an increase in intracellular $\mathrm{Ca}^{2+}$ concentration in well defined areas of the dendritic tree. The deeper interneurons, particularly the basket cells, strongly inhibit firing of specific Purkinje cells located laterally to the 
basket cells. It is possible that effective inhibition of the distal Purkinje cell dendrites requires the collaborative effort of a group of neurons, particularly when an elaborate structure such as the Purkinje cells dendrites has to be modulated and the $\mathrm{Ca}^{2+}$ spike evoked by the powerful climbing fiber input has to be inhibited. The rather weak coupling enables a synchronized activity in a relatively wide time window. Interestingly, this time window is well within the duration of the climbing fiber response. That is, this weak synchrony may allow group of inhibitory interneurons to coordinate their activity to achieve effective inhibition of the distal dendrites of the Purkinje cell.

This report adds to a growing body of evidence showing that electrotonic coupling is more common in the CNS than previously realized (Bouskila and Dudek, 1993; O'Donnell and Grace, 1993; Draguhn et al., 1998). Contrary to the classical view, we have demonstrated here that electrical synapses and intrinsic currents interact to form a highly modifiable communication pathway. A small modulation of membrane potential or membrane resistivity, either by neuromodulators or by spontaneous synaptic activity, can strongly modulate the strength of electrotonic coupling as well as its interaction with intrinsic currents. This enables a tight control on the strength and the time window of the synchronized activity.

\section{REFERENCES}

Alonso A, Khateb A, Fort P, Jones BE, Mühlethaler M (1996) Differential oscillatory properties of cholinergic and non-cholinergic nucleus basalis neurons in guinea pig brain slice. Eur J Neurosci 8:169-182.

Benardo LS (1997) Recruitment of GABAergic inhibition and synchronization of inhibitory interneurons in rat neocortex. J Neurophysiol 77:3134-3144.

Bourrat F, Sotelo C (1983) Postnatal development of the inferior olivary complex in the rat. 1. An electron microscopic study of the medial accessory olive. Dev Brain Res 8:291-310.

Bouskila Y, Dudek FE (1993) Neuronal synchronization without calcium-dependent synaptic transmission in the hypothalamus. Proc Natl Acad Sci USA 90:3207-3210.

Buzsáki G, Chrobak JJ (1995) Temporal structure in spatially organized neuronal ensembles: a role for interneuronal networks. Curr Opin Neurobiol 5:504-510.

Callaway JC, Lasser-Ross N, Ross WN (1995) IPSPs strongly inhibit climbing fiber-activated $\left[\mathrm{Ca}^{2+}\right]_{\mathrm{i}}$ increases in the dendrites of cerebellar Purkinje neurons. J Neurosci 15:2777-2787.

De Schutter E, Bower JM (1994) An active membrane model of the cerebellar Purkinje cell II. Simulation of synaptic responses. J Neurophysiol 71:401-419.

Deschênes M, Madariaga-Domich A, Steriade M (1985) Dendrodendritic synapses in the cat reticularis thalami nucleus: a structural basis for thalamic spindle synchronization. Brain Res 334:165-168.

Dickson CT, Alonso A (1997) Muscarinic induction of synchronous population activity in the entorhinal cortex. J Neurosci 17:6729-6744.
Draguhn A, Traub RD, Schmitz D, Jefferys JGR (1998) Electrical coupling underlies high-frequency oscillations in the hippocampus in vitro. Nature 394:189-192.

Eccles JC, Ito M, Szentágothai J (1967) The cerebellum as a neuronal machine, pp 93-115. New York: Springer.

Gutnick MJ, Lobel-Yaakov R, Rimon G (1985) Incidence of neuronal dye-coupling in neocortical slices depend on the plane of section. Neuroscience 15:659-666.

Häusser M, Clark BA (1997) Tonic synaptic inhibition modulates neuronal output pattern and spatiotemporal synaptic integration. Neuron 19:665-678.

Ito M (1984) The cerebellum and neural control, pp 21-39. New York: Raven.

Kondo S, Marty A (1998) Differential effects of noradrenaline on evoked, spontaneous and miniature IPSCs in rat cerebellar stellate cells. J Physiol (Lond) 509:233-243.

Llano I, Gerschenfeld HM (1993) Inhibitory synaptic currents in stellate cells of rat cerebellar slices. J Physiol (Lond) 468:177-200.

Logan SD, Pickering AE, Gibson IC, Nolan MF, Spanswick D (1996) Electrotonic coupling between rat sympathetic preganglionic neurons in vitro. J Physiol (Lond) 495:491-502.

Marr D (1969) A theory of cerebellar cortex. J Physiol (Lond) 202:437-470.

Michelson HB, Wong RKS (1994) Synchronization of inhibitory neurons in the guinea-pig hippocampus in vitro. J Physiol (Lond) 477:35-45.

O’Donnell P, Grace AA (1993) Dopaminergic modulation of dye coupling between neurons in the core and shell regions of the nucleus accumbens. J Neurosci 13:3456-3471.

Palay SL, Chan-Palay V (1974) Cerebellar cortex, cytology and organization, pp 180-233. Berlin: Springer).

Pouzat C, Hestrin S (1997) Developmental regulation of basket/stellate cell $\rightarrow$ Purkinje cell synapses in the cerebellum. J Neurosci 17:9104-9112.

Rinzel J, Terman D, Wang X-J, Ermentrout B (1998) Propagating activity pattern in large-scale inhibitory neuronal networks. Science 279:1351-1355.

Sotelo C, Llinás R (1972) Specialized membrane junctions between neurons in the vertebrate cerebellar cortex. J Cell Biol 53:271-289.

Sultan F, Bower JM (1998) Quantitative Golgi study of the rat Cerebellar molecular layer interneurons using principal component analysis. J Comp Neurol 393:353-373.

Traub RD (1995) Model of synchronized population bursts in electrically coupled interneurons containing active dendritic conductances. J Comp Neurosci 2:283-289.

Van Vreeswijk C, Abbott LF (1994) When inhibition not excitation synchronizes neural firing. J Comp Neurosci 1:313-321.

Vincent P, Marty A (1996) Fluctuations of inhibitory postsynaptic currents in Purkinje cells from rat cerebellar slices. J Physiol (Lond) 494:183-199.

Wang X-J, Buzsáki G (1996) Gamma oscillation by synaptic inhibition in a Hippocampal interneuronal network model. J Neurosci 16: 6402-6413.

Whittington MA, Traub RD, Jefferys JGR (1995) Synchronized oscillations in interneuron networks driven by metabotropic glutamate receptor activation. Nature 373:612-615. 